\title{
Validation of the WHO-defined $20 \%$ circulating blasts threshold for diagnosis of leukemic transformation in primary myelofibrosis
}

\author{
Mythri Mudireddy ${ }^{1}$, Naseema Gangat ${ }^{1}$, Curtis A. Hanson ${ }^{2}$, Rhett P. Ketterling ${ }^{3}$, Animesh Pardanani ${ }^{1}$ and Ayalew Tefferi ${ }^{1}$
}

Leukemic transformation is the most dreaded complication in patients with myeloproliferative neoplasms (MPN); overall risk is estimated at $14 \%$ for primary myelofibrosis (PMF), 7\% for polycythemia vera (PV) and $4 \%$ for essential thrombocythemia $(E T)^{1}$. The dismal prognosis of blast phase MPN (MPN-BP) was recently underlined in a large Mayo Clinic study of 248 informative patients and further validated in a separate cohort of 162 cases recruited from multiple Italian institutions ${ }^{2}$. Current treatment approaches for MPN-BP, including allogeneic hematopoietic stem cell transplant $(\mathrm{HCT})$, are largely ineffective in securing long-term survival ${ }^{2}$. On the other hand, HCT has been shown to produce long-term remissions in $\sim 50 \%$ of patients with myelofibrosis, if undertaken before blast transformation ${ }^{3}$. At present, diagnosis of MPN-BP employs the World Health Organization (WHO)-defined criteria for acute myeloid leukemia (AML): presence of $\geq 20 \%$ blasts either in the bone marrow $(\mathrm{BM})$ or in the peripheral blood $(\mathrm{PB})^{4}$. In other words, neither scenario requires compartmental concordance. However, it is currently not clear if this $20 \%$ WHO threshold for circulating blasts is valid in the context of primary myelofibrosis (PMF), especially in terms of its prognostic equivalency to that of $\mathrm{BM}$ blast percentage-defined blast phase PMF (PMF-BP) and its distinction from accelerated phase disease (PMF-AP),

\footnotetext{
Correspondence: Ayalew Tefferi (tefferi.ayalew@mayo.edu)

${ }^{1}$ Department of Internal Medicine and Laboratory Medicine, Division of Hematology, Mayo Clinic, Rochester, MN, USA

${ }^{2}$ Department of Internal Medicine and Laboratory Medicine, Division of Hematopathology, Mayo Clinic, Rochester, MN, USA

Full list of author information is available at the end of the article
}

operationally defined by the presence of $10-19 \%$ circulating blasts ${ }^{5}$.

The current study was approved by the Mayo Clinic institutional review board. Diagnosis of PMF and PMF-BP were according to WHO criteria ${ }^{4}$. Designation of unfavorable karyotype was according to previously published criteria $^{6}$. Study patients were recruited from institutional databases of chronic phase or blast phase PMF. As per the study design, phenotypic and prognostic comparisons considered four distinct categories of PMF patients: (i) PMF with 5-9\% circulating blasts, (ii) PMF-AP with $10-19 \%$ circulating blasts, (iii) PMF-BP with $\geq 20 \%$ BM blasts, regardless of $\mathrm{PB}$ blast percentage (i.e., BM-defined PMF-BP), and (iv) PMF-BP with $\geq 20 \%$ PB blasts but $<20 \%$ BM blasts (i.e., PB-defined PMF-BP). Statistical analyses for chronic phase PMF considered clinical and laboratory data collected at the time of documented PB blast count of $\geq 5 \%$ and for PMF-BP, date of leukemic transformation. Survival was computed from the date of either leukemic transformation (for PMF-BP) or documentation of the increased blast threshold for PMF-AP and PMF with 5-9\% circulating blasts. Non-parametric statistics was used to determine significance of differences among groups, in the distribution of continuous or nominal variables. The Stat View (SAS Institute, Cary, NC, USA) statistical package was used for all calculations.

The total number of study patients was 144 and included 41 patients with chronic phase PMF and 5-19\% circulating blasts and 103 with PMF-BP (Table 1); the 41 patients with chronic phase PMF included 28 patients with 5-9\% circulating blasts and 13 patients with $10-19 \%$ circulating blasts (i.e., PMF-AP); the 103 patients with PMF-BP included 71 patients in whom diagnosis was 
Table 1 Comparison of clinical and laboratory parameters between "blast phase primary myelofibrosis (PMF-BP)" and "chronic phase primary myelofibrosis with 5-19\% circulating blasts"

\begin{tabular}{|c|c|c|c|c|c|c|}
\hline Variables & $\begin{array}{l}\text { All patients } \\
(n=144)\end{array}$ & $\begin{array}{l}\text { PMF-BP with } \geq 20 \% \text { BM } \\
\text { blasts }(n=71)\end{array}$ & $\begin{array}{l}\text { PMF-BP with }<20 \% \text { BM } \\
\text { blasts but } \geq 20 \% \text { PB blasts } \\
\text { ( } n=32 \text { ) }\end{array}$ & $\begin{array}{l}\text { PMF-AP with } \\
10-19 \% \text { PB blasts } \\
(n=13)\end{array}$ & $\begin{array}{l}\text { PMF with } 5-9 \% \text { PB blasts } \\
(n=28)\end{array}$ & $\begin{array}{l}P \\
\text { value }\end{array}$ \\
\hline Age in years; median (range) & $68(43-89)$ & $68(44-87)$ & $66(44-84)$ & $64(46-80)$ & $69(43-89)$ & 0.6 \\
\hline Age $>65$ years; $n(\%)$ & $81(56 \%)$ & $41(58 \%)$ & $16(50 \%)$ & $6(46 \%)$ & $18(64 \%)$ & 0.6 \\
\hline Sex (male); $n(\%)$ & $96(67 \%)$ & $49(69 \%)$ & $23(72 \%)$ & 7 (54\%) & $17(61 \%)$ & 0.6 \\
\hline Transfusion dependent; $n$ (\%) & $68(48 \%)$ & $31(44 \%)$ & $13(42 \%)$ & 7 (54\%) & $17(61 \%)$ & 0.4 \\
\hline Hemoglobin, g/dl; median (range) & $9.3(6.1-13.7)$ & $9.1(6.1-13.7)$ & $8.9(6.3-11.2)$ & $10(7.0-11.6)$ & $9.7(6.6-13.5)$ & 0.88 \\
\hline Platelets, $\times 10^{9} / L$; median (range) & 79 (4-984) & $67(4-568)$ & $79(6-670)$ & $139(24-885)$ & $157(14-984)$ & 0.004 \\
\hline Leukocytes, $\times 10^{9} / \mathrm{L}$; median (range) & $21(0.5-219)$ & $22.3(0.5-208.4)$ & $19.5(3.0-139.5)$ & $21.5(2.1-75)$ & $20.1(1.8-219)$ & 0.9 \\
\hline $\begin{array}{l}\text { Karyotype "N" evaluable }=119 \\
(83 \%)\end{array}$ & & & & & & 0.03 \\
\hline Favorable; $n(\%)$ & $64(54 \%)$ & $31(51 \%)$ & 7 (39\%) & $5(38 \%)$ & $21(78 \%)$ & \\
\hline Unfavorable; $n(\%)$ & $55(46 \%)$ & $30(49 \%)(\mathrm{N}$ evaluable $=61)$ & $11(61 \%)(\mathrm{N}$ evaluable = 18) & $8(62 \%)$ & $6(22 \%)(\mathrm{N}$ evaluable $=27)$ & \\
\hline $\begin{array}{l}\text { Karyotype "N" evaluable }=119 \\
(83 \%)\end{array}$ & & & & & & 0.02 \\
\hline Normal; $n(\%)$ & $35(29 \%)$ & $16(26 \%)$ & $2(11 \%)$ & $3(23 \%)$ & $14(52 \%)$ & \\
\hline Abnormal; $n$ (\%) & $84(71 \%)$ & $45(74 \%)(\mathrm{N}$ evaluable $=61)$ & $16(89 \%)(\mathrm{N}$ evaluable $=18)$ & $10(77 \%)$ & $13(48 \%)(\mathrm{N}$ evaluable $=27)$ & \\
\hline $\begin{array}{l}\text { Driver mutation status "N" } \\
\text { evaluable }=113(78 \%)\end{array}$ & & & & & & 0.3 \\
\hline$J A K 2 ; n(\%)$ & $67(59 \%)$ & $32(67 \%)$ & $12(50 \%)$ & $8(62 \%)$ & $15(53 \%)$ & \\
\hline CALR Type $1 /$ like; $n$ (\%) & $18(16 \%)$ & $9(19 \%)$ & $4(17 \%$ & $2(15 \%)$ & $3(11 \%)$ & \\
\hline CALR Type 2/like; $n(\%)$ & $9(8 \%)$ & $1(2 \%)$ & $2(8 \%)$ & $3(23 \%)$ & $3(11 \%)$ & \\
\hline$M P L ; n(\%)$ & $6(5 \%)$ & $2(4 \%)$ & $1(4 \%)$ & $0(0 \%)$ & $3(11 \%)$ & \\
\hline Triple-negative; $n$ (\%) & $13(12 \%)$ & $4(8 \%)(\mathrm{N}$ evaluable $=48)$ & $5(21 \%)(\mathrm{N}$ evaluable $=24)$ & $0(0 \%)$ & $4(14 \%)$ & \\
\hline
\end{tabular}

The values in bold indicate a significant $p$-value $<0.05$

$B M$ bone marrow, $P B$ peripheral blood, PMF primary myelofibrosis, PMF-BP blast phase PMF, PMF-AP accelerated phase PMF with $10-19 \%$ circulating blasts, JAK2 Janus kinase 2, CALR Calreticulin, MPL MPL proto-oncogene

confirmed by the demonstration of $\geq 20 \%$ BM blasts and 32 patients in whom BM examination revealed $<20 \%$ blasts but diagnosis of PMF-BP was based on the presence of $\geq 20 \%$ PB blasts. These four operational groups of patients with PMF displayed similar age $(p=0.6)$ and gender $(p=0.6)$ distribution and were also similar in their need for red cell transfusions $(p=0.4)$, hemoglobin level $(p=0.9)$, leukocyte count $(p=0.9)$, and driver mutational status $(p=0.3)$, including the incidence of type $1 /$ like CALR mutations ( $11 \%$ vs $15 \%$ vs $17 \%$ vs $19 \%$, respectively) (Table 1). On the other hand, platelet counts were significantly lower in both BM-defined (median $67 \times 10^{9} / \mathrm{l}$ ) and PB-defined (median $79 \times 10^{9} / \mathrm{l}$ ) PMF-BP, compared to those seen in PMF patients with $5-9 \%$ circulating blasts $\left(\right.$ median $\left.157 \times 10^{9} / \mathrm{l}\right)$ or PMF-AP (median $\left.139 \times 10^{9} / \mathrm{l}\right)(p$ $=0.004$ ). Significant differences were also noted for karyotype, as detailed in Table 1 , and the incidences of abnormal $(p=0.02)$ and unfavorable $(p=0.03)$ karyotype were the highest in patients with PB-defined PMF-BP and lowest in PMF with 5-9\% circulating blasts.

Most importantly, survival data of BM-defined PMF-BP ( $\geq 20 \%$ BM blasts) were indistinguishable from those of PB-defined PMF-BP ( $<20 \%$ BM blasts but $\geq 20 \%$ PB blasts) (HR 1.1; 95\% CI 0.7-1.6; $p=0.8$; Fig. 1), whereas survival in both instances was significantly shorter than that of patients with chronic phase PMF with 5-19\% circulating blasts: HRs (95\% CI) were 2.6 (1.7-3.8) for PMF-BP (both BM- and PB-defined combined) vs PMF with 5-19\% circulating blasts; 2.9 (1.8-4.7) for BM-defined PMF-BP vs PMF with 5-9\% circulating blasts; 2.7 (1.5-4.8) for PBdefined PMF-BP vs PMF with 5-9\% circulating blasts; 2.1 (1.2-3.8) for BM-defined PMF-BP vs PMF-AP; and 2.0 (1.0-3.9) for PB-defined PMF-BP vs PMF-AP (Fig. 1). Survival was not significantly different between PMF-AP and PMF with 5-9\% circulating blasts (HR 1.4, 95\% CI 0.7-2.9; $p=0.3$; Fig. 1).

The observations from the current study confirm the prognostic validity of the current WHO criteria for defining leukemic transformation in PMF and the appropriateness, in this regard, of utilizing PB blast percentage, irrespective of BM blast content. The particular issue carries significant importance for practice because of the difficulty in obtaining adequate tissue and accurately quantifying blast content in the BM of patients with PMF, especially during accelerated and blast phase disease. Immunostaining of BM biopsy specimens for CD34 is unlikely to resolve the issue because CD34 also stains megakaryocytes, endothelial cells, and is also broadly expressed in myeloid progenitors in PMF that do not necessarily meet the morphologic criteria for blasts. Furthermore, the presence of significant bone marrow fibrosis and osteosclerosis makes it that much harder to 


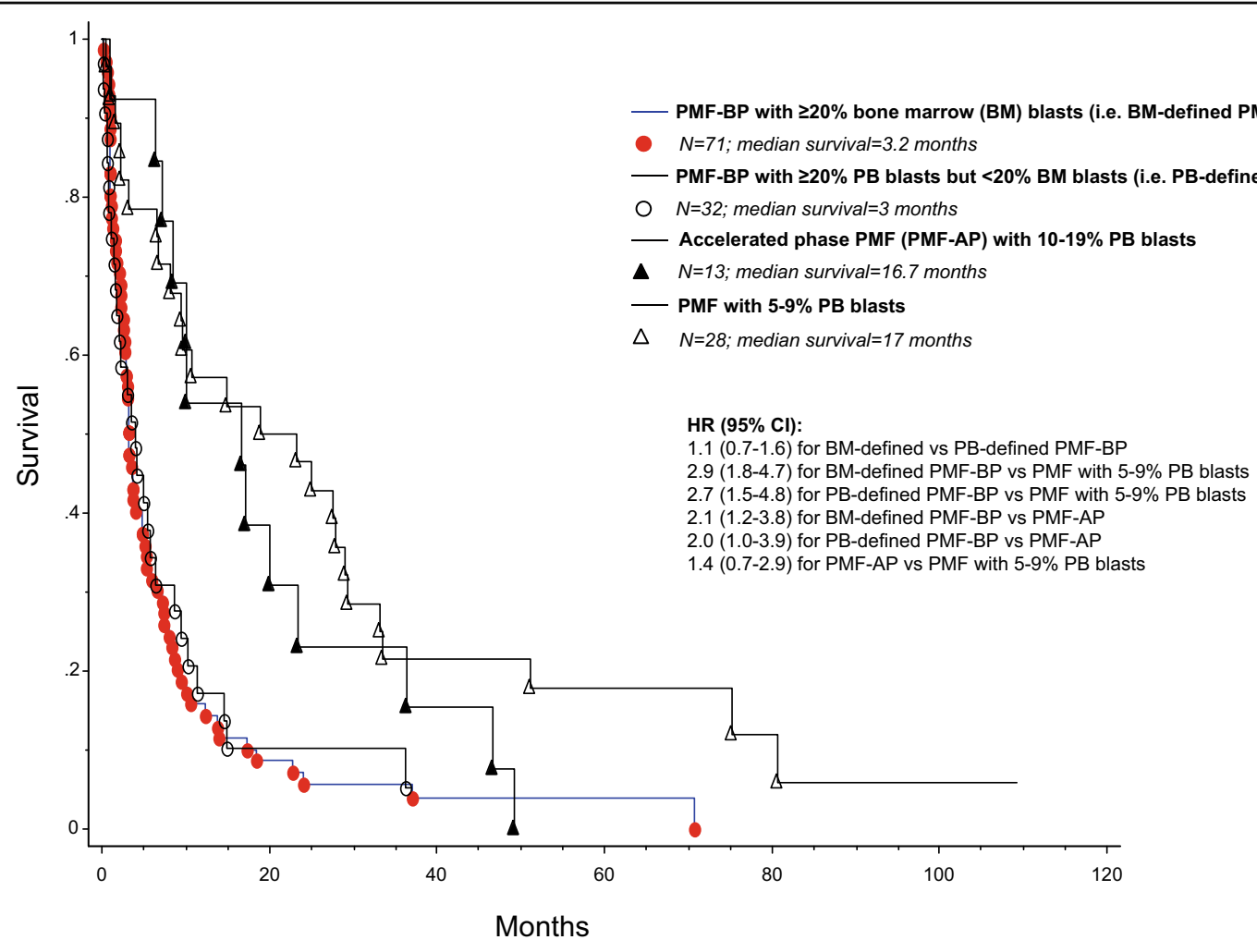

Fig. 1 Overall survival of 144 patients with either "chronic phase primary myelofibrosis with 5-19\% peripheral blood (PB) blasts" or "blast phase primary myelofibrosis (PMF-BP)"

accurately estimate BM blast count. The current study was not designed to address the issue of accelerated phase PMF and whether or not the presence of excess blasts, in otherwise chronic phase PMF, carries prognostic relevance that is independent of current prognostic models ${ }^{7}$.

\section{Author details}

'Department of Internal Medicine and Laboratory Medicine, Division of Hematology, Mayo Clinic, Rochester, MN, USA. ²Department of Internal Medicine and Laboratory Medicine, Division of Hematopathology, Mayo Clinic, Rochester, MN, USA. ${ }^{3}$ Department of Internal Medicine and Laboratory Medicine, Division of Laboratory Genetics and Genomics, Mayo Clinic, Rochester, MN, USA

\section{Conflict of interest}

The authors declare that they have no conflict of interest.

\section{Publisher's note}

Springer Nature remains neutral with regard to jurisdictional claims in published maps and institutional affiliations.
Received: 16 February 2018 Accepted: 9 April 2018.

Published online: xx xxx 2018

\section{References}

1. Tefferi, A. et al. Long-term survival and blast transformation in molecularly annotated essential thrombocythemia, polycythemia vera, and myelofibrosis. Blood 124, 2507-2513 (2014).

2. Tefferi, A. et al. Blast phase myeloproliferative neoplasm: Mayo-AGIMM study of 410 patients from two separate cohorts. Leukemia 32, 1200-1210 (2018).

3. Samuelson Bannow, B. T. et al. Hematopoietic Cell Transplantation for Myelofibrosis: the Dynamic International Prognostic Scoring System Plus Risk Predicts Post-Transplant Outcomes. Biol. Blood Marrow Transplant. 24, 386-392 (2017).

4. Swerdlow, S. H. et al. WHO Classification of Tumours of Haematopoietic and Lymphoid Tissues. (IARC, Lyon, France, 2017).

5. Tefferi, A. et al. Predictors of greater than $80 \%$ 2-year mortality in primary myelofibrosis: a Mayo Clinic study of 884 karyotypically annotated patients. Blood 118, 4595-4598 (2011).

6. Gangat, N. et al. DIPSS plus: a refined Dynamic International Prognostic Scoring System for primary myelofibrosis that incorporates prognostic information from karyotype, platelet count, and transfusion status. J. Clin. Oncol. 29, 392-397 (2011).

7. Guglielmelli, P. et al. MIPSS70: Mutation-Enhanced International Prognostic Score System for Transplantation-Age Patients With Primary Myelofibrosis. J. Clin. Oncol. 36, 310-318 (2017). 